\title{
Magnitude and antimicrobial susceptibility patterns of extended-spectrum $\beta$-lactamase producing Enterobacteriaceae recovered from environmental samples at Tikur Anbessa Specialized Hospital, Ethiopia
}

Shemse Sebre Muktar ( $\nabla$ shemsesebre@gmail.com )

Addis Ababa University College of Health Sciences

Woldaregay Erku

Addis Ababa University School of Medicine

Wude Mihret

Armauer Hansen Research Institute

Aminu Seman

Addis Ababa University School of Medicine

Tewachw Awoke

Bahir Dar University College of Medical and Health Sciences

\section{Zelalem Desalegn}

Addis Ababa University School of Medicine

Adane Mihret

Addis Ababa University School of Medicine

Tamrat Abebe

Addis Ababa University School of Medicine

\section{Research article}

Keywords: Enterobacteriaceae, ESBL, inanimate hospital environment, antimicrobial susceptibility

Posted Date: July 22nd, 2020

DOI: https://doi.org/10.21203/rs.3.rs-44084/v1

License: (c) (i) This work is licensed under a Creative Commons Attribution 4.0 International License. Read Full License 


\section{Abstract \\ Background}

The hospital environment especially the intensive care units (ICUs) contributes to the spread of extended-spectrum $\beta$ lactamase (ESBL) producing Enterobacteriaceae, which are contributing to increasing morbidity and mortality rates. We aimed to assess the rate of environmental contamination of ESBL producing Enterobacteriaceae and their antimicrobial susceptibility patterns.

\section{Methods}

A Cross-sectional study was conducted at Tikur Anbessa Specialized Hospital (TASH) from June to July, 2018. A total of 65 environmental swab were taken from inanimate items. All isolates from the inanimate environment were cultured by using ESBL ChromoSelect Agar and identified using conventional bacteriological methods. All ESBL producing Enterobacteriaceae isolates were tested for their antimicrobial susceptibility using the disc diffusion method according to the methods and interpretive criteria of the Clinical and Laboratory Standards Institute (CLSI).

\section{Results}

From a total of 65 environmental swab samples, 16 (24.6\%) were ESBL producing Enterobacteriaceae. The most predominant ESBL producing isolates were E. coli (8/16, 50\%) and Klebsiella spp (6/16, 37.6\%). The Pediatrics and Medical-Surgical ICU exhibited the highest ESBL producing Enterobacteriaceae, each with $(37.5 \%, 6 / 16)$. Linens and ventilators were associated with high rates of ESBL isolates positivity each with equal $31.3 \%$ detection rate. ESBL producing Enterobacteriaceae isolates had significantly high resistance level to ampicillin (100\%), ceftazidime (93.8\%), cefepime (87.5\%) and cefotaxime (86.7\%). However, low resistance level was recorded against amikacin (18.8\%).

\section{Conclusion}

The appearance of ESBL producing Enterobacteriaceae among ICUs hospital environment is posing a serious threat for the control of nosocomial infections. The high resistance found from this study shows the need for policies for devising infection control procedures and screening policies for ESBL on a routine basis.

\section{Background}

In Gram-negative bacteria, $\beta$-lactamase production remains the most important mechanism of resistance to $\beta$-lactam group of antibiotics and has become a global challenge in infection control. One group of these enzymes is called extended-spectrum $\beta$-lactamases (ESBLs) [1-3]. ESBLs hydrolyze a wide range of cephalosporins including the oxyimino group of cephalosporins such as ceftriaxone, ceftazidime, cefotaxime and the monobactam drugs such as aztreonam, but do not hydrolyze cephamycins and carbapenems, and their activity is inhibited by clavulanic acid [3, 4].

ESBL producing bacteria have been detected in inanimate surfaces of hospitals which could contribute to their dissemination $[5,6]$. The detection of bacterial pathogens including ESBL producing bacteria from inanimate hospital environments is due to an innate ability of bacteria to survive on surfaces for long periods [7]. For instance, Klebsiella spp and Escherichia coli, which are the predominant ESBL producing Enterobacteriaceae, have been reported to survive up to 30 and 16 months, respectively, on inanimate dry surfaces [5]. 
In Ethiopia the rate of ESBLs on clinical isolates has been increased from time to time [8-10]. The increased rate of ESBLproducing bacteria in Ethiopia may be due to the cross transmission of strains from patient to patient or from the inanimate hospital environment to the patients and from health care workers to patients and vice versa [6]. Moreover, the horizontal transmission of resistance genes through genetic mobile elements has also important role in adaption of bacteria for various conditions in the hospital environments [11, 12]. The contaminated hospital environment in the transmission of ESBL producing Enterobacteriaceae is widely considered the principal cause of hospital acquired infections (HAls) [13].

Despite the recent worldwide spread of ESBL in K. pneumoniae and E. coli isolates from HAls, their dissemination has been little studied especially on inanimate hospital environments in Ethiopia. This study was therefore aimed at determining the rate of environmental contamination of ESBL producing Enterobacteriaceae and their antimicrobial susceptibility patterns will have a valuable input to control the emerging problem in this direction.

\section{Methods}

\section{Study design and Environmental Sampling}

This cross-sectional study included 65 environmental swabs at Tikur Anbessa Specialized Hospital from June to July, 2018. The samples were collected from four sites including the intensive care units of Surgical, Pediatric, Medical and Medical-Surgical wards of the hospital. The samples were collected on a single occasion, using pre-moistened sterile swabs from environmental surfaces and medical equipment including beds, monitors, linens, ventilators, suction machine, Lobby (furniture), work station and sinks in accordance to suggestions from previous studies $[14,15]$.

\section{Culture and Bacterial isolates}

The swab samples were enriched overnight at $37^{\circ} \mathrm{C}$ in brain heart infusion broth (BHI) (Merck, Germany) [16]. A loop full of bacterial growth was transferred to ESBL ChromoSelect Agar (SIGMA-ALDRICH) and incubated for $24 \mathrm{~h}$ at $37^{\circ} \mathrm{C}$ to assess the ESBL production. Bacterial identification was achieved using specific colony color on ESBL ChromoSelect agar and Gram staining combined with panel of biochemical tests including Triple Sugar Iron agar (TSI), urea, citrate, Sulfide Indole Motility (SIM) medium, Lysine Iron Agar (LIA), mannitol, malonate and oxidase [17].

\section{Antimicrobial susceptibility testing}

Antimicrobial susceptibilities of ESBL producing Enterobacteriaceae isolates were determined using disc diffusion method on Mueller-Hinton agar (MHA) (Oxoid, UK) based on Clinical and Laboratory Standards Institute (CLSI) performance standard methods and interpretive criteria [18]. Isolates were tested for susceptibility to the following 13 antibiotics (in $\mu \mathrm{g} /$ disk): ampicillin (10), amoxicillin/ clavulanic acid (20/10), ceftriaxone (30), ceftazidime (30), cefotaxime (30), cefepime (30), aztreonam (30), meropenem (10), gentamicin (10), amikacin (30), ciprofloxacin (5), trimethoprim/ sulfamethoxazole (1.25/23.75) and chloramphenicol (30). Data analysis was performed using SPSS version 25 software program.

\section{Phenotypic confirmation of ESBL}

All the identified isolates were phenotypically confirmed for ESBL production using Combination Disk (CD) method on Mueller-Hinton Agar (MHA) (Oxoid, UK). Briefly, a cefotaxime disc $(30 \mu \mathrm{g})$ was placed $20 \mathrm{~mm}$ away from a disc containing cefotaxime-clavulanic acid $(30 / 10 \mu \mathrm{g})$. When the inhibition zone between at least one of the combination discs and its 
corresponding single antibiotic disc differed by $\geq 5 \mathrm{~mm}$, the strain was identified as an ESBL producer according CLSI guideline [18]. Escherichia coli ATCC 25922 and Klebsiella pneumonia ATCC 700603 were used as the control strains.

MDR was defined as acquired non-susceptibility to at least one agent in three or more antimicrobial categories [19].

\section{Ethics approval and consent to participate}

The study protocol was reviewed and approved by Department of Microbiology, Immunology and Parasitology Research Ethics Review Committee (DRERC), College of Health Sciences, Addis Ababa University.

\section{Results}

\section{Culture results and Bacterial profiles}

ESBL producing Enterobacteriaceae were isolated in 16 of the $65(24.6 \%)$ environmental samples, with Escherichia coli $(8 / 16,50 \%)$, Klebsiella pneumoniae and Klebsiella oxytoca each with $(3 / 16,18.8 \%)$ being the most predominant ESBL producing environmental isolates (Table 1).

Table 1: Frequency of ESBL producing bacteria from inanimate environments at TASH, 2018.

\begin{tabular}{lrr}
\hline Isolates & Frequency & Percentage (\%) \\
\hline Klebsiella pneumoniae & 3 & 18.8 \\
Escherichia coli & 8 & 50 \\
Enterobacter spp & 2 & 12.5 \\
Klebsiella oxytoca & 3 & 18.8 \\
Total & 16 & 100 \\
\hline
\end{tabular}

\section{Distribution of ESBL producing Enterobacteriaceae across different ICUs}

The Pediatrics and Medical-Surgical ICU exhibited the highest ESBL producing Enterobacteriaceae, each with (37.5\%, $6 / 16)$. The major pathogens in these ICUs were E. coli, $(37.5 \%, 3 / 8)$. Surgical and Medical ICUs were contaminated with Klebsiella pneumoniae as high as $(33.3 \%, 1 / 3)$ (Table 2$)$.

Table 2: Distribution ESBL producing Enterobacteriaceae across different ICUs at TASH, 2018

\begin{tabular}{|c|c|c|c|c|}
\hline \multirow{4}{*}{ Isolates } & \multicolumn{4}{|c|}{ Sites of environmental samples in the ICUs } \\
\hline & Surgical & Pediatrics & Medical & Medical-Surgical N=15 \\
\hline & $\mathrm{N}=15$ & $\mathrm{~N}=17$ & $\mathrm{~N}=18$ & $\mathrm{n}(\%)$ \\
\hline & n (\%) & n $(\%)$ & $\mathrm{n}(\%)$ & \\
\hline Klebsiella pneumoniae & $1(6.7)$ & $1(5.9)$ & $1(5.6)$ & \\
\hline E. coli & $1(6.7)$ & $3(17.6)$ & $1(5.6)$ & $3(20)$ \\
\hline Enterobacter spp & & & & 2(13.3) \\
\hline Klebsiella oxytoca & & 2(11.8) & & $1(6.7)$ \\
\hline Total $(n=16)$ & 2(12.5) & $6(37.5)$ & $2(12.5)$ & $6(37.5)$ \\
\hline
\end{tabular}




\section{Distribution of ESBL producing Enterobacteriaceae over different surfaces}

The highest number of ESBL producing bacteria were obtained from linens and ventilators each, with 5(31.3\%) followed by monitor and bed, each with 2(12.5\%) (Table 3).

Table 3: Distribution of ESBL producing Enterobacteriaceae over different surfaces at TASH, 2018

\begin{tabular}{|c|c|c|c|c|c|}
\hline \multirow{2}{*}{ Sites } & \multicolumn{5}{|c|}{ Isolates n (\%) } \\
\hline & Klebsiella pneumoniae & E. coli & Enterobacter spp & Klebsiella oxytoca & Total $=16$ \\
\hline Monitor & & 1 & 1 & & $2(12.5)$ \\
\hline Sink & & & 1 & & $1(6.3)$ \\
\hline Bed & & 2 & & & $2(12.5)$ \\
\hline Ventilator & 2 & 2 & & 1 & $5(31.3)$ \\
\hline Linens & 1 & 2 & & 2 & $5(31.3)$ \\
\hline lobby (furniture) & & 1 & & & $1(6.3)$ \\
\hline Total $=16$ & $3(18.8)$ & $8(50)$ & $2(12.5)$ & $3(18.8)$ & $16(100)$ \\
\hline
\end{tabular}

\section{Antimicrobial susceptibility pattern of ESBL}

The Extended spectrum $\beta$ lactamase (ESBL) producing Enterobacteriaceae isolates obtained from this study were found to show significantly high resistance level to ampicillin (100\%), ceftazidime (93.8\%), cefepime (87.5\%), azetronome (81.3\%), and cefotaxime (86.7\%) and amoxicillin with clavulanic acid (81.3\%). Low resistance level was recorded for amikacin (18.8\%) followed by gentamicin and meropenem each with (37.5\%). Among the most common isolated ESBL, $E$. coli showed a higher rate of resistance to ampicillin (100\%), ceftazidime (87.5\%), ceftriaxone (87.5\%) and cefotaxime (87.5\%). Furthermore, non-beta lactam antimicrobials such as ciprofloxacin and sulfamethoxazole-trimethoprim resistance was observed in $37.5 \%$ and $75 \%$ of $E$. coli isolates, respectively. K. pneumoniae revealed high resistance (100\%) to ampicillin, ceftriaxone, ceftazidime, cefepime, amoxicillin with clavulanic acid and sulfamethoxazole-trimethoprim (Table 4). 
Table 4: Antimicrobial susceptibility pattern of ESBL producing bacteria from inanimate environments at TASH, 2018.

\begin{tabular}{|c|c|c|c|c|c|c|c|c|c|c|c|c|c|c|}
\hline \multirow[b]{2}{*}{ Isolates } & \multirow[b]{2}{*}{ Ptn } & \multicolumn{13}{|c|}{ Antimicrobial agent's n (\%) } \\
\hline & & AMP & AZM & CTX & CRO & CTZ & FEP & AMC & CHL & MRP & AK & GEN & CIP & SXT \\
\hline \multirow[t]{2}{*}{ K. pneumoniae } & $\mathrm{R}$ & $3(100)$ & $2(66.7)$ & $2(66.7)$ & $3(100)$ & $3(100)$ & $3(100)$ & $3(100)$ & $1(33.3)$ & $2(66.7)$ & 1(33.3) & $1(33.3)$ & $1(33.3)$ & $3(100)$ \\
\hline & $\mathrm{S}$ & $0(0)$ & $1(33.3)$ & 1(33.3) & $0(0)$ & $0(0)$ & $0(0)$ & $0(0)$ & $2(66.7)$ & $1(33.3)$ & $2(66.7)$ & $2(66.7)$ & $2(66.7)$ & $0(0)$ \\
\hline \multirow[t]{2}{*}{ E. coli } & $\mathrm{R}$ & $8(100)$ & $6(75)$ & $7(87.5)$ & $7(87.5)$ & $7(87.5)$ & $6(75)$ & $5(62.5)$ & $3(37.5)$ & $2(25)$ & $1(12.5)$ & $3(37.5)$ & $3(37.5)$ & $6(75)$ \\
\hline & $\mathrm{S}$ & $0(0)$ & $2(25)$ & $1(12.5)$ & $1(12.5)$ & $1(12.5)$ & $2(25)$ & $3(37.5)$ & $5(62.5)$ & $6(75)$ & $7(87.5)$ & $5(62.5)$ & $5(62.5)$ & $2(25)$ \\
\hline \multirow[t]{2}{*}{ Enterobacter spp } & $\mathrm{R}$ & $2(100)$ & $2(100)$ & $2(100)$ & $2(100)$ & $2(100)$ & $2(100)$ & $2(100)$ & $2(100)$ & $1(50)$ & $0(0)$ & $1(50)$ & $2(100)$ & $2(100)$ \\
\hline & S & $0(0)$ & $0(0)$ & $0(0)$ & $0(0)$ & $0(0)$ & $0(0)$ & $0(0)$ & $0(0)$ & $1(50)$ & $2(100)$ & $1(50)$ & $0(0)$ & $0(0)$ \\
\hline \multirow[t]{2}{*}{ K. oxytoca } & $\mathrm{R}$ & $3(100)$ & $3(100)$ & $3(100)$ & $3(100)$ & $3(100)$ & $3(100)$ & $3(100)$ & $2(66.7)$ & 1(33.3) & 1(33.3) & $1(33.3)$ & $2(66.7)$ & $2(66.7)$ \\
\hline & S & $0(0)$ & $0(0)$ & $0(0)$ & $0(0)$ & $0(0)$ & $0(0)$ & $0(0)$ & $1(33.3)$ & $2(66.7)$ & $2(66.7)$ & $2(66.7)$ & 1(33.3) & $1(33.3)$ \\
\hline \multirow[t]{2}{*}{ Total } & $\mathrm{R}$ & $8(100)$ & $13(81.3)$ & $14(86.7)$ & $15(93.8)$ & $15(93.8)$ & $14(87.5)$ & $15(81.3)$ & $8(50)$ & $6(37.5)$ & $3(18.8)$ & $6(37.5)$ & $8(50)$ & 13(81.3) \\
\hline & S & $0(0)$ & $3(18.7)$ & $2(13.3)$ & $1(6.3)$ & $1(6.3)$ & $2(12.5)$ & 1(18.7) & $8(50)$ & $10(62.5)$ & $13(81.3)$ & $10(62.5)$ & $8(50)$ & $3(18.8)$ \\
\hline
\end{tabular}

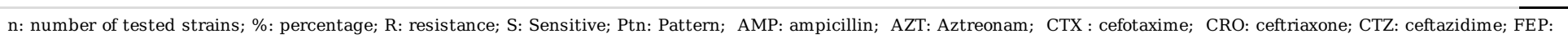

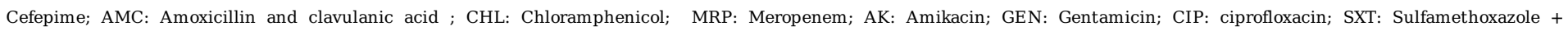
trimethoprim

\section{Distribution of MDR phenotypes}

Of the total 16 ESBL producing Enterobacteriaceae isolates, 15(93.8\%) were resistant to at least 3 antibiotics and twelve multi-drug resistance phenotypes were detected. The predominant MDR phenotype was observed in E. coli (8/8, 100\%), Klebsiella pneumonia $(3 / 3,100 \%)$ and Enterobacter spp $(2 / 2,100 \%)$. The most common MDR combinations found among E. coli isolates was against 7 classes of antibiotics (Table 5).

Table 5: Multidrug-resistance profiles of ESBL producing Enterobacteriaceae at TASH, 2018.

\begin{tabular}{|c|c|}
\hline MDR phenotype & Frequency, n (\%) \\
\hline \multicolumn{2}{|l|}{ E. coli } \\
\hline AMP, CTX, AMC, MRP, AK, CIP, SXT & $2(25)$ \\
\hline AMP, CTX, SXT & $2(25)$ \\
\hline AMP, CTX, AMC & $2(25)$ \\
\hline AMP, CTX, AMC, AK, CIP, CHL, SXT & $1(12.5)$ \\
\hline AMP, CTX, AMC, SXT & $1(12.5)$ \\
\hline Total & $8 / 8(100)$ \\
\hline \multicolumn{2}{|l|}{ Klebsiella pneumonia } \\
\hline AMP, CTX, AMC, GEN, SXT & $1(33.3)$ \\
\hline AMP, CTX, AMC, SXT & $1(33.3)$ \\
\hline AMP, CTX, AMC, MRP, AK, CIP, SXT & $1(33.3)$ \\
\hline Total & $3 / 3(100)$ \\
\hline \multicolumn{2}{|l|}{ Klebsiella oxytoca } \\
\hline AMP, CTX, AMC, MRP, AK, CIP, STX & $1(33.3)$ \\
\hline AMP, CTX, AMC, AK, CIP, CHL, SXT & $1(33.3)$ \\
\hline Total & $2 / 3(66.7)$ \\
\hline \multicolumn{2}{|l|}{ Enterobacter spp } \\
\hline AMP, CTX, AMC, MRP, AK, CIP, SXT & $1(50)$ \\
\hline AMP, CTX, AMC, GEN, CIP, SXT & $1(50)$ \\
\hline Total & $2(100)$ \\
\hline
\end{tabular}

AMP: ampicillin; CTX : cefotaxime; AMC: Amoxicillin and clavulanic acid ; CHL: Chloramphenicol; MRP: Meropenem; Sulfamethoxazole + trimethoprim, MDR: Multi Drug Resistant

\section{Discussion}


In our study, ESBL producing Enterobacteriaceae were isolated in 16 of the 65 (24.6\%) environmental samples. In published reports, the percentage of inanimate hospital environments contaminated with ESBL are significantly varied ranging from $3 \%$ to $33 \%$ of surfaces in patients' rooms on regular hospitals wards, as reported from Gonder in Ethiopia (14.8\%) [6], Tunisia (4\%) [11], Israel (9\%) [20], Tunisia (4\%) [5], UK (3.1\%) [12] and Pakistan (33\%) [2]. These discrepancies could possibly be due to differences in patient colonization load, hospital's cleaning/disinfection protocols, study design including timing of sample collection and laboratory method used [5, 11, 20, 21].

We found a high proportion of ESBL producers among E. coli and $K$. pneumoniae isolates which has similarly been reported from studies in Gondar, Ethiopia [6], Zimbabwe [22], Gaza in Palestine [23] and Algeria [24]. Given the fact that Klebsiella spp and Escherichia coli have capacity to survive up to 30 and 16 months, respectively, on inanimate dry surfaces [5]. Moreover, isolation of such Enterobacteriaceae is highly indicative of fecal contamination and poor hand washing practices amongst health workers and patients [25].

The most prevalent ESBL producer detected in the current study was E. coli (50\%) as compared to K. pneumoniae (18.8\%) which has similarly been reported from studies in Gaza, Palestine (E. coli $40 \%$ vs K. pneumoniae $20 \%$ ) [23]. In contrast to our result, different findings from most reports in other parts of the world ESBL production was more predominantly found among Klebsiella spp isolates: Zimbabwe (Klebsiella spp $60 \%$ vs E. coli 40\%) [22], Algeria (K. pneumoniae $48.5 \%$ vs E. coli $22.8 \%$ ) [24], Gondar, Ethiopia (K. pneumoniae 42.1\% vs E. coli 35.1\%) [6], Sudan (K. pneumoniae 37\% vs E. coli (0\%) [26] and on clinical isolates from another study in Ethiopia (K. pneumoniae $72.7 \%$ vs E. coli $22.7 \%$ ) [9].

The Pediatrics and Medical-Surgical ICU exhibited the highest ESBL producing Enterobacteriaceae, each with (37.5\%, 6/16). This may lead to cross-infections between patients in the same ICU because patients present in these ICUs are likely to have reduced immune system due to illness, surgical and mechanical manipulation, and/or the use of immunesuppressors and other therapeutic drugs, all of which increasing patients susceptibility to infections [27, 28].

Bed linens, ventilators, beds and sink were inanimate surfaces observed to be associated with variable degree of ESBL producing Enterobacteriaceae positivity, with $31.3 \%, 12.5 \%$ and $6.5 \%$, respectively. The identification of ESBL producing bacteria on linens, sink, bed and other environmental surface is consistent with reports from the literatures $[5,6,12,20]$. It is generally assumed that ESBL producing Gram-negative bacteria require moist or damp sites for enhanced longevity [12, 24]. Moreover, bed linens are considered to be high patient-contact surfaces and therefore the detected pathogens might have been shed by the infected/colonized patients occupying the particular beds.

In regards to level of antimicrobial resistance among our ESBL producing isolates, significantly high resistance level was recorded to penicillin groups such as ampicillin (100\%) and cephalosporin groups such as cefuroxime (96\%), ceftazidime (92\%), cefepime (86\%), azetronome (80\%) and cefotaxime (72\%). These results were mostly comparable with results from other studies conducted in Ethiopia (cefpirome, cefpodoxime, ceftazidime, ceftriaxone and amoxicillin with clavulanic acid each with $100 \%$ resistance level) [6] and Algeria where ampicillin resistance level was reported at $98.1 \%$ [24]. Probably lack of antibiotic resistance screening and confirmatory testing prior to using these drugs could be responsible for accumulation of such high level of resistance among ESBL producing bacteria in the hospital environments.

High resistance rate was also recorded form ESBL-producing isolates for non-beta-lactam antimicrobials such as, cotrimoxazole (81.3\%), ciprofloxacin and chloramphenicol each with (50\%). This was in close agreement with studies done in Ethiopia (ciprofloxacin 56\%) [6], from another study in Addis Ababa from clinical isolates (cotrimoxazole 77\%, ciprofloxacin 46.3\%) [8]; Tikur Anbessa Specialized Hospital, from clinical isolates, cotrimoxazole (83.6\%) and Chloramphenicol (61.8\%) [9]. The most active drugs for ESBL-producing isolates were amikacin (81.2\%). Low resistance rate of amikacin in our study could be explained by the dearth in usage of amikacin in our study setting.

Reports from other studies showed that multidrug resistance (MDR) to the commonly prescribed antimicrobial agents are more common among ESBL producers found from inanimate hospital surfaces [29,30]. Hence, unwise use of antibiotics 
can inadvertently favor emergence of multidrug resistant bacterial strains $[11,28]$. The finding from the current study showed that $(15 / 16,93.8 \%)$ of the ESBL producing bacterial isolates were resistant to at least 3 antibiotics, showing how rampant MDR Enterobacteriaceae are in the hospital environments. This finding is higher than reports from Northwest Ethiopia (75\%) [31], Zimbabwe (75\%) [22] and Iran (79.4\%) [32].

\section{Conclusions}

In this study the appearance of ESBL producing Enterobacteriaceae among ICUs hospital environment in the study site poses a serious threat for the control of nosocomial infections. Occurrence of multidrug resistance (MDR) to the commonly prescribed antimicrobial agents is found to be more common among ESBL producers, showing the need for policies to prevent misuse and overuse of antimicrobial agents. Moreover, it also indicates the need for infection control procedures and screening policies for ESBL on a routine basis in the hospital environment.

\section{Abbreviations}

TASH: Tikur Anbessa specialized Hospital; ICUs: Intensive care units; ESBL: Extended-spectrum $\beta$-lactamase: CD: combination disk; \%: Percent; ${ }^{\circ} \mathrm{C}$ : Degree Celsius; ATCC: American Type Culture Collection; CLSI: Clinical Laboratory Standards Institute; HAls: Hospital Acquired Infections; MDR: Multi Drug Resistant; DRERC: Department of Microbiology, Immunology and Parasitology Research Ethics Review Committee; TSI: Triple Sugar Iron agar, SIM: Sulfide Indole Motility medium, LIA: Lysine Iron Agar, MHA: Mueller-Hinton Agar

\section{Declarations}

\section{Ethics approval and consent to participate}

The study protocol was approved by the Department of Microbiology, Immunology and Parasitology Research Ethics Review Committee (DRERC), College of Health Sciences, Addis Ababa University (Ref. no. DRERC/17/18/02-G). Prior to sample collection, written approval was obtained from administrative unit of Tikur Anbessa Specialized Hospital. However, consent was not obtained because it was inanimate environmental samples.

\section{Consent for publication}

Not applicable.

\section{Availability of data and materials}

The dataset supporting the findings of this article is available from the corresponding author upon request.

\section{Competing interests}

The authors declare that they have no competing interests.

\section{Funding}

This research work were funded by Addis Ababa University and Armauer Hansen Research Institute.

\section{Author Contributions}

All authors have read and approved the manuscript.

\section{Acknowledgements}


The authors here by thank Addis Ababa University and Armauer Hansen Research Institute for their financial and material support and Tikur Anbessa Specialized Hospital staff.

\section{References}

1. Chroma M, Kolar M. Genetic methods for detection of antibiotic resistance: focus on extended-spectrum $\beta$ lactamases. Biomed Pap Med Fac Univ Palacky Olomouc Czech Repub. 2010;154(4):289-96.

2. Bukhari A, Arshad M, Raza S, Azam M, Mohsin M. Emergence of extended spectrum beta-lactamases-producing strains belonging to cefotaxime-M-1 class from intensive care units patients and environmental surfaces in Pakistan. Int J One Health. 2016;2:69-74.

3. Kumar CS. Molecular Mechanism of Drug Resistance: Common Themes. Drug Resistance in Bacteria, Fungi. Malaria: Springer; 2017. pp. 25-46.

4. Laudy AE, Rog P, Smolińska-Król K, Ćmiel M, Słoczyńska A, Patzer J, et al. Prevalence of ESBL-producing Pseudomonas aeruginosa isolates in Warsaw, Poland, detected by various phenotypic and genotypic methods. PloS one. 2017;12(6).

5. Guet-Revillet H, Le Monnier A, Breton N, Descamps P, Lecuyer H, Alaabouche I, et al. Environmental contamination with extended-spectrum $\beta$-lactamases: is there any difference between Escherichia coli and Klebsiella spp? Am. J Infect Control. 2012;40(9):845-8.

6. Engda T, Moges F, Gelaw A, Eshete S, Mekonnen F. Prevalence and antimicrobial susceptibility patterns of extended spectrum beta-lactamase producing Entrobacteriaceae in the University of Gondar Referral Hospital environments, northwest Ethiopia. BMC Res Notes. 2018;11(1):335.

7. Chemaly RF, Simmons S, Dale C Jr, Ghantoji SS, Rodriguez M, Gubb J, et al. The role of the healthcare environment in the spread of multidrug-resistant organisms: update on current best practices for containment. THER ADV INFECT DIS. 2014;2(3-4):79-90.

8. Teklu DS, Negeri AA, Legese MH, Bedada TL, Woldemariam HK, Tullu KD. Extended-spectrum beta-lactamase production and multi-drug resistance among Enterobacteriaceae isolated in Addis Ababa, Ethiopia. Antimicrob Resist Infect Control. 2019;8(1):39.

9. Legese MH, Weldearegay GM, Asrat D. Extended-spectrum beta-lactamase-and carbapenemase-producing Enterobacteriaceae among Ethiopian children. Infect Drug Resist. 2017;10:27.

10. Desta K, Woldeamanuel Y, Azazh A, Mohammod H, Desalegn D, Shimelis D, et al. High gastrointestinal colonization rate with extended-Spectrum $\beta$-lactamase-producing Enterobacteriaceae in hospitalized patients: emergence of Carbapenemase-Producing K. pneumoniae in Ethiopia. PloS one. 2016;11(8).

11. Dziri R, Klibi N, Alonso CA, Jouini A, Ben Said L, Chairat S, et al. Detection of CTX-M-15-producing Escherichia coli isolates of lineages ST131-B2 and ST167-A in environmental samples of a Tunisian hospital. Microb Drug Resist. 2016;22(5):399-403.

12. Muzslay M, Moore G, Alhussaini N, Wilson A. ESBL-producing Gram-negative organisms in the healthcare environment as a source of genetic material for resistance in human infections. J Hosp Infect. 2017;95(1):59-64.

13. Facciolà A, Pellicanò G, Visalli G, Paolucci I, Venanzi Rullo E, Ceccarelli M, et al. The role of the hospital environment in the healthcare-associated infections: a general review of the literature. Eur Rev Med Pharmacol Sci. 2019;23(3):1266-78.

14. Rocha IV, Xavier DE, Almeida KRHd O, SRd, Leal NC. Multidrug-resistant Acinetobacter baumannii clones persist on hospital inanimate surfaces. Braz J Infect Dis. 2018;22(5):438-41.

15. Chen C-H, Lin Y-L, Chen K-H, Chen W-P, Chen Z-F, Kuo H-Y, et al. Bacterial diversity among four healthcare-associated institutes in Taiwan. Sci Rep. 2017;7(1):1-11. 
16. Shamsizadeh Z, Nikaeen M, Esfahani BN, Mirhoseini SH, Hatamzadeh M, Hassanzadeh A. Detection of antibiotic resistant Acinetobacter baumannii in various hospital environments: potential sources for transmission of Acinetobacter infections. Environ Health Prev Med. 2017;22(1):44.

17. Garcia LS. Clinical microbiology procedures handbook: Amer Soc Microbiology Press. 2010.

18. CLSI. Performance standards for antimicrobial susceptibility testing. 28th ed. CLSI supplement M100. Wayne, PA: Clinical and Laboratory Standards Institute; 2018.

19. Magiorakos AP, Srinivasan A, Carey R, Carmeli Y, Falagas M, Giske C, et al. Multidrug-resistant, extensively drugresistant and pandrug-resistant bacteria: an international expert proposal for interim standard definitions for acquired resistance. Clin Microbiol Infect. 2012;18(3):268-81.

20. D’Agata EM, Venkataraman L, DeGirolami P, Samore M. Molecular epidemiology of ceftazidime-resistant gramnegative bacilli on inanimate surfaces and their role in cross-transmission during nonoutbreak periods. J Clin Microbiol. 1999;37(9):3065-7.

21. Freeman JT, Nimmo J, Gregory E, Tiong A, De Almeida M, McAuliffe GN, et al. Predictors of hospital surface contamination with Extended-spectrum $\beta$-lactamase-producing Escherichia coli and Klebsiella pneumoniae: patient and organism factors. Antimicrob Resist Infect Control. 2014;3(1):5.

22. Mbanga J, Sibanda A, Rubayah S, Buwerimwe F, Mambodza K. Multi-Drug Resistant (MDR) Bacterial Isolates on Close Contact Surfaces and Health Care Workers in Intensive Care Units of a Tertiary Hospital in Bulawayo, Zimbabwe. J Adv Med Med Res. 2018:1-15.

23. Al Laham NA. Distribution and antimicrobial resistance pattern of bacteria isolated from operation theaters at Gaza strip. Journal of Al Azhar University-Gaza (Natural Sciences). 2012;14(1):19-34.

24. Manel D, Abdelbasset M, Houria C. Prevalence and characterization of extended-spectrum $\beta$-lactamase-producing Enterobacteriaceae isolated from hospital environments. Asian J Microbiol Biotechnol Environ Sci. 2014;16(2):19-27.

25. Sserwadda I, Lukenge M, Mwambi B, Mboowa G, Walusimbi A, Segujja F. Microbial contaminants isolated from items and work surfaces in the post-operative ward at Kawolo general hospital, Uganda. BMC Infect Dis. 2018;18(1):68.

26. Nurain AM, Bilal NE, Ibrahim ME. The frequency and antimicrobial resistance patterns of nosocomial pathogens recovered from cancer patients and hospital environments. Asian Pac J Trop Biomed. 2015;5(12):1055-9.

27. Abreu AC, Tavares RR, Borges A, Mergulhão F, Simões M. Current and emergent strategies for disinfection of hospital environments. J Antimicrob Chemother. 2013;68(12):2718-32.

28. Yusuf B, Okwong OK, Mohammed A, Abubakar KS, Babayo A, Barma MM, et al. Bacterial contamination of intensive care units at a tertiary hospital in Bauchi, Northeastern Nigeria. Am J Intern Med. 2017;5(3):46.

29. Zenati K, Sahli F, Garcia V, Bakour S, Belhadi D, Rolain JM, et al. Occurrence and clonal diversity of multidrug-resistant Klebsiella pneumoniae recovered from inanimate surfaces in Algerian hospital environment: First report of armA, qnrB and aac(6')-lb-cr genes. J Glob Antimicrob Resist. 2017;10:148-53.

30. Dziri R, Klibi N, Alonso CA, Said LB, Bellaaj R, Slama KB, et al. Characterization of extended-spectrum beta-lactamase (ESBL)-producing Klebsiella, Enterobacter, and Citrobacter obtained in environmental samples of a Tunisian hospital. Diagn Microbiol Infect Dis. 2016;86(2):190-3.

31. Getachew H, Derbie A, Mekonnen D. Surfaces and air bacteriology of selected wards at a referral hospital, Northwest Ethiopia: a cross-sectional study. Int. J. Microbiol. 2018;2018.

32. Tajeddin E, Rashidan M, Razaghi M, Javadi SS, Sherafat SJ, Alebouyeh M, et al. The role of the intensive care unit environment and health-care workers in the transmission of bacteria associated with hospital acquired infections. $J$ Infect Public Health. 2016;9(1):13-23. 